Dieses Dokument ist eine Zweitveröffentlichung von:

Arnold Jacobshagen

Oper als szenischer Text.

Louis Paliantis Inszenierungsanweisungen zu Meyerbeers Le Prophète

Veröffentlicht in:

Giacomo Meyerbeer, Le Prophète. Edition, Konzeption, Rezeption, hrsg. von Matthias Brzoska, Andreas Jacob und Nicole K. Strohmann, S. 181-212. Verlag: Olms | Jahr: 2009

Bereitgestellt von musiconn.publish

DOI: https://doi.org/10.25366/2021.54

Lizenz: CC BY-NC-ND 4.0, https://creativecommons.org/licenses/bync-nd/4.0/deed.de 


\title{
Oper als szenischer Text: \\ Louis Paliantis Inszenierungsanweisungen zu Meyerbeers Le Prophète
}

\author{
Arnold Jacobshagen, Köln
}

Die Erkenntnis, dass die Oper ein multimediales Kunstwerk ist, dessen individueller Werkcharakter sich nicht allein auf die schriftliche Fixierung der Partitur oder des Librettos reduzieren lässt, hat in jüngerer Zeit das Interesse der musikwissenschaftlichen Forschung in zunehmendem Maße auch auf die Quellen zu den Uraufführungsinszenierungen von Opern vergangener Epochen gerichtet. Im Bereich der Edition sind besonders weit reichende Konsequenzen hieraus im Rahmen der Rossini-Gesamtausgabe gezogen worden. So umfasst die Ausgabe von Rossinis letzter Oper Guillaume Tell neben der vierbändigen Partitur und einem kritischen Bericht auch eine Edition des Librettos in verschiedenen Fassungen, eine Edition des vermutlich originalen Pariser Regiebuchs sowie einen separaten Band zur Ikonografie, der die bildlichen Inszenierungsdokumente der Pariser Premiere des Jahres I 829 enthält. ${ }^{1}$ Die editorische Ausweitung auf äußerst heterogene Text- und Bildgattungen im Rahmen einer Komponisten-Gesamtausgabe offenbart ein Dilemma, das sich für die Opernedition nur ausnahmsweise stellt: Gewöhnlich wird a priori die Partitur als alleinige Trägerin der Textsubstanz angesehen. Zudem ist die Edition weiterer Primärquellen sehr aufwendig, sof olche überhaupt überliefert sind.

Die historische Aufführungspraxis von Opern des 19. Jahrhunderts, die allmählich auch die Werke Wagners und Verdis zu erreichen beginnt, konzentriert sich daher weiterhin primär auf die Interpretation des Notentextes. Doch seit dem 19. Jahrhundert wurde auch die Inszenierung in zunehmendem Maße als integraler Werkaspekt angesehen, dessen Individualität sich materiell möglichst in der vollständigen Neuanfertigung sämtlicher Ausstattungsmerkmale nach den Vorgaben eines einheitlichen künstlerischen Konzepts konkreti-

Edizione critica delle opere di Gioachino Rossini, I/39: Guillaume Tell, hg. von M. Elizabeth C. Bartlet, I-IV (Partitura), V (Commento critico), VI (Testi), Pesaro: Fondazione Rossini 1992; Guillaume Tell di Gioachino Rossini: fonti iconografiche, hg. von M. Elizabeth C. Bartlet, Pesaro: Fondazione Rossini 1996. 
sierte. Welch entscheidende Rolle die Inszenierung etwa in Richard Wagners Konzeption des Gesamtkunstwerks zukommt, ist seit langem unumstritten; wie sehr aber beispielsweise auch Giuseppe Verdi seit den I860er-Jahren auf sämtliche Inszenierungsdetails persönlich Einfluss nahm, haben erst jüngere Forschungen eindrucksvoll deutlich gemacht. ${ }^{2}$ Sowohl Wagners als auch Verdis intensive Bestrebungen um eine vollständige Kontrolle aller musikalischen wie auch szenischen Aspekte ihrer Opern haben historisch eine gemeinsame Basis: beide Komponisten wurden nachhaltig geprägt durch die jahrzehntelange Auseinandersetzung mit der Pariser Grand Opéra, jener Form des musikalischen Dramas, in der sich zuerst ein modernes Verständnis von Operninszenierung entwickelte und die zugleich auch über die besten finanziellen, personellen und bühnentechnischen Voraussetzungen hierfür verfügte.

Mit den so genannten Livrets de mise en scène besitzen wir für die Pariser Oper zudem eine besondere Quellengattung szenischer Texte, deren Relevanz für die historische Rekonstruktion und die musikdramaturgische Analyse in der Forschung längst anerkannt ist. Unter diesen Regiebüchern nimmt die rund 200 Titel umfassende Collection de mises en scène de Grands Opéras et d'Opéras-Comiques représentés pour la première fois à Paris, rédigées et publiées par M. L. Palianti einen zentralen Platz ein. Unter allen Regiebüchern Paliantis ist dasjenige zu Giacomo Meyerbeers Le Prophète (1849) bei weitem das umfangreichste und detailreichste. Die Gründe hierfür sind vielfältig; sie hängen mit der außerordentlichen Komplexität des Werkes ebenso zusammen wie mit den neuartigen inszenatorischen Mitteln, unter denen neben der Verwendung von Rollschuhen für die Darsteller des so genannten "Schlittschuhballetts « vor allem die berühmte elektrische »Prophetensonne « zu nennen ist.

Historisch weitaus bedeutsamer aber als die im Prophète exponierten Requisiten und technischen Erfindungen dürfte die Tatsache sein, dass hier der Komponist selbst die treibende Kraft hinter der Festlegung und verbindlichen Überlieferung aller inszenatorischen Details gewesen ist. Eine These des vorliegenden Beitrags lautet daher, dass mit Meyerbeers Prophète der bislang erste umfassend dokumentierte Beleg dafür vorliegt, dass ein Opernkomponist die letzte Kontrolle über die verschiedenen Aspekte der musikalischen wie auch der szenischen Realisierung seines Werkes für sich reklamierte, und zwar nicht

Vgl. zuletzt u.a. La Realizzazione Scenica dello Spettacolo Verdiano. Atti del Congresso internazionale di studi, hg. von Pierluigi Petrobelli und Fabrizio Della Seta Parma, Parma: Istituto nazionale di studi verdiani 1996; sowie Olga Jesurum, »L'aspetto visivo delle opere di Verdi: le interpretazioni scenografiche della prima metà del Novecento in Italia«, in: Verdi $200 I$. Atti del convegno internazionale, Parma/New York/New Haven, hg. von Fabrizio Della Seta, Firenze: Olschki 2003, 339-349. 
nur für die Premiere, sondern darüber hinaus nach Möglichkeit auch für spätere Inszenierungen und Aufführungen andernorts.

Um sich die Bedeutung dieses Umstandes zu vergegenwärtigen, bedarf es zunächst der Erörterung einiger Voraussetzungen. Im ersten Teil des vorliegenden Beitrags werde ich mich mit der Pariser Inszenierungspraxis zur Zeit Meyerbeers auseinandersetzen und dabei den in zahlreichen jüngeren Untersuchungen genährten Mythos der »originalen « Inszenierung etwas zu relativieren versuchen. Sodann werde ich im zweiten Teil auf einige Aspekte und Beispiele aus Louis Paliantis Inszenierungsprotokoll zu Meyerbeers Le Prophète näher eingehen. Und abschließend werde ich mich mit den Quellen auseinandersetzen, die uns über die konkrete Zusammenarbeit zwischen Meyerbeer und Palianti zur Verfügung stehen.

Die Pariser Livrets de mise en scène sind mittlerweile als eine der Opernforschung vertraute Quellengattung zu bezeichnen. Seit den späten I970erJahren lenkte H. Robert Cohen das Interesse der Musikwissenschaft auf die Regiebücher der Pariser Oper, die handschriftlich oder im Druck vervielfältigt wurden und in erster Linie dazu dienten, die Theater in der Provinz und im Ausland so genau wie möglich über die Pariser Aufführungen zu informieren. ${ }^{3}$ Zwei Anthologien mit insgesamt 22 vollständig faksimilierten Inszenierungsprotokollen hat Cohen inzwischen vorgelegt, ${ }^{4}$ sowie - gemeinsam mit MarieOdile Gigou - einen Katalog der Sammlung der Regiebücher in der Bibliothèque de l'Association de la Régie Thêâtrale in Paris.' 199I fasste Cohen seine Erkenntnisse dahingehend zusammen, dass die Operninszenierung im

3 H. Robert Cohen, »On the Reconstruction of the Visual Elements of French Grand opéra", in: International Musicological Society. Report of the Twelfth Congress Berkeley 1977, hg. von Daniel Heartz and Bonnie Wade, Kassel/Basel/London: Bärenreiter I98I, 463-480; id., "La Conservation de la tradition scénique sur la scène lyrique en France au XIX ${ }^{\mathrm{e}}$ siècle», in: Revue de Musicologie 64 (1978), 253-268; id., "Le rôle de l'Association des Régisseurs de Théâtre dans la conservation et la communication de la mise en scène», in: Revue de la société d'histoire du théâtre 33 (1985), $425 \mathrm{ff}$; id., »On preparing critical studies of the original smise en scène< of nineteenth-century operas «, in: Opera E Libretto II, hg. von Gianfranco Folena, Maria Teresa Muraro und Giovanni Morelli, Firenze: Olschki I993, 2 I 5-224.

4 H. Robert Cohen, The Original Staging Manuals for Twelve Parisian Operatic Premières, Stuyvesant/New York: Pendragon Press I991; H. Robert Cohen, The Original Staging Manuals for Ten Parisian Operatic Premières, Stuyvesant/New York I998.

s Cent ans de mise en scène lyrique en France (environ 1830-1930). Catalogue descriptif des livrets de mise en scène, des libretti annotés et des partitions annotées dans la Bibliothèque de l'Association de la Régie Théâtrale (Paris), hg. von H. Robert Cohen und Marie-Odile Gigou, Stuyvesant/New York: Pendragon Press 1986. 
I9. Jahrhundert durch die fortwährende und unveränderte Konservierung der originalen Mise en scène gekennzeichnet gewesen sei:

»Staging in Paris and the French provinces throughout the nineteenth century and well into the twentieth was an art of preservation rather than creation. Régisseurs strove to conserve, to the extent possible, the original mise en scène of an opera's premiere as transcribed in the production book. Staging, in a word, was not intended to be altered. ${ }^{6}$

Jüngere Forschungen zur Pariser Oper, etwa von Rebecca Susan Wilberg, Karin Pendle, Stephen Wilkins und Cormack Newark, basieren bereits auf Cohens Annahmen und heben die normative Bedeutung der Regiebücher dementsprechend hervor. Wilberg zufolge beruhe der Wert dieser Publikationen

»auf der im i9. Jahrhundert gültigen Ästhetik, das gesamte Werk in allen seinen Facetten - Musik, Libretto und Inszenierung - als Teil eines integralen Ganzen zu begreifen. Man ging davon aus, dass das Bühnenbild und die Positionen der Darsteller auf der Bühne auch in allen späteren Produktionen genauestens befolgt würden, sowohl an der [Pariser] Opéra selbst wie auch bei auswärtigen Aufführungen. «7

Diese Hypothese lässt sich aufgrund der Quellenlage nur schwer verifizieren. Cohen übersah, dass etliche der von ihm als originale Regiebücher der Pariser Uraufführungen in Faksimile-Editionen publizierten Dokumente wesentlich späteren Datums sind und mit den Pariser Premieren nicht immer in unmittelbarer Beziehung stehen. Bereits vor einiger Zeit habe ich die These von der Perpetuierung originaler Inszenierungen am Beispiel von Aubers Fra Diavolo zu relativieren versucht. Ausgehend von der Beobachtung, dass das von Cohen in einer Faksimile-Edition vorgelegte, angeblich originale Dokument keineswegs aus dem Uraufführungsjahr i 830 stammen konnte (u.a. da der Autor des Hefts Louis Palianti damals erst neunzehn Jahre alt war und in der Fußzeile auf den erst I 846 gegründeten Verlag Brandus verwiesen wird), habe ich nach Auswertung zahlreicher weiterer Pariser Inszenierungszeugnisse festgestellt, dass Fra Diavolo während seiner mehr als 800 Aufführungen an der OpéraComique im i9. Jahrhundert auch im Bereich der Inszenierung ständigen Veränderungen unterworfen war. ${ }^{8}$

6 Cohen, The Original Staging Manuals for Twelve Parisian Operatic Premières, xxiii.

7 Rebecca Susan Wilberg, The "mise en scène" at the Paris Opéra, Salle Le Peletier (I82I-1873) and the staging of the first French "Grand opera«: Meyerbeer's "Robert le Diable«, Diss. Brigham Young University, Ann Arbor 1990, 9.

8 Arnold Jacobshagen, "Staging at the Opéra-Comique in nineteenth-century Paris. Auber's Fra Diavolo and the livrets de mise en scène", in: Cambridge Opera Journal I 3/3 (200I), 239-260; vgl. dazu auch Isabelle Moindrot, "Les mises en scène du Prophète au delà de la première parisienne ", siehe im vorliegenden Band Seite 525. 
Noch aufschlussreicher ist der Fall von Paliantis Inszenierungsanweisungen zu Halévys La Juive. ${ }^{9}$

\section{Collection de Mises en Scène, rédigées et publiées par M. L. PALIANTI. Propriété pour tous pays. - Réimprestiona ou traductions interdites. \\ LA

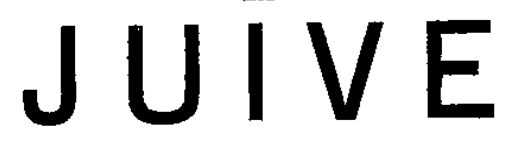

OPERA EN CINQ ACTES

De M. E. S C R I B E

Repreśenté pour la première fois à Paris, sur le Théatre de l'opérd, le 23 fêvrier 1835.

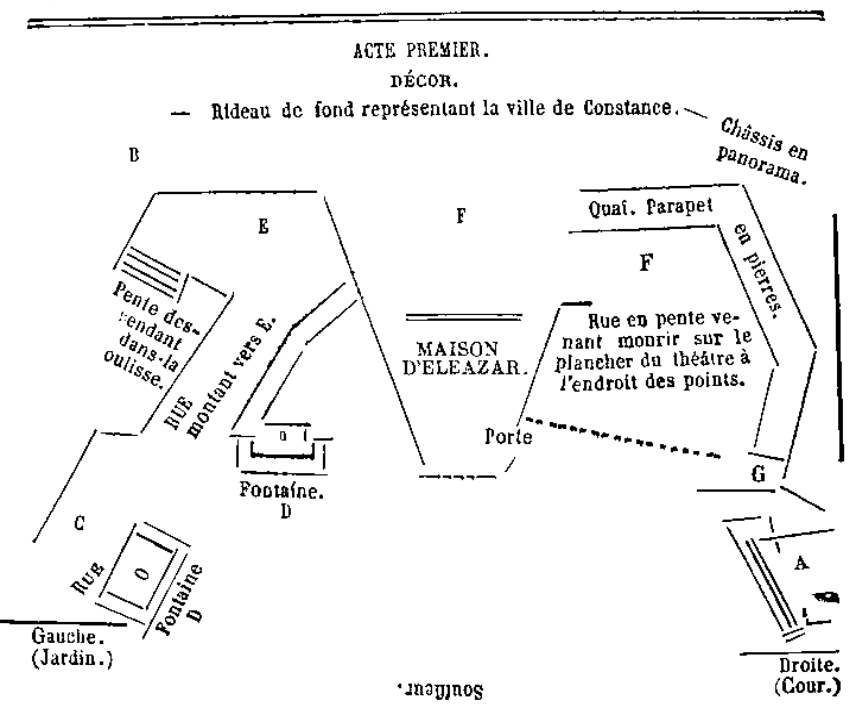
Un carrefour de la ville de Constance en 1414. - A droite A, le portail d'une église.
on y monte par six ou buit marches de pierre. - La maison d'Eleazar, à l'angle d'une rue, fait face au public. - Oa y entre par le eóté. - D. Fontaine.

INTRODUGTION. Au lever du rideau les portes de l'église sont ouvertes. - Quelques groupes de femmea
occupent les premiers plang.-Quelques-unes sont assises sur les marches do l'église.-

Abb. I: Louis Palianti, Mise en scène, La Juive von Fromental Halévy

9 Collection de Mises en Scène, rédigées et publiées par M. L. PALIANTI. / Propriété pour tous pays. - Réimpressions ou traductions interdites. / LA / JUIVE / OPERA EN CINQ ACTES / De M. E. SCRIBE / MusiQue DE F. HAlevY / Représenté pour la première fois à Paris, sur le Théâtre de l'Opéra, le 23 février I 835 . Reprint in Cohen, The Original Staging Manuals for Twelve Parisian Operatic Premières, I37-I 50. 
Karin Pendle und Stephen Wilkins, die sich intensiv mit diesem ebenfalls von Cohen publizierten Dokument auseinandergesetzt haben, behaupten explizit, es sei I 835 gedruckt worden. ${ }^{10}$ Cormac Newark hat vor einiger Zeit versucht, diese Quelle noch genauer zu datieren und ist dabei zu der Einschätzung gelangt, sie sei »kurz nach der letzten Kostümprobe« entstanden, wegen des »Fehlens der ursprünglich geplanten ersten beiden Szenen des III. Aktes, die noch vor der Premiere gestrichen wurden ${ }^{11}{ }^{11}$

In Wirklichkeit aber wurde dieses Dokument erst rund drei Jahrzehnte nach der Premiere von La Juive veröffentlicht. ${ }^{12}$ Der Urheberrechtsvermerk «Propriété pour tous pays. - Réimpressions ou traductions interdites » reflektiert nämlich die Urheberrechtslage der I 860er-Jahre, und das Pflichtexemplar dieser Broschüre kam erst im Jahre 1870 in die Bibliothèque Nationale. Inzwischen habe ich eine von Louis Duverger tatsächlich unmittelbar nach der Premiere in Umlauf gebrachte Mise en scène untersucht, die sich ohne Frage auf die originale Inszenierung von Charles Edmond Duponchel und Adolphe Nourrit bezieht, und mit Paliantis Anweisungen verglichen. ${ }^{13}$

10 Karin Pendle/Stephen Wilkins, »Paradise Found. The Salle le Peletier and French Grand Opera «, in: Opera in Context. Essays on Historical Staging from the Late Renaissance to the Time of Puccini, edited by Mark A. Radice, Portland/Oregon: Amadeus Press 1998, I7I-207: 190, note 60.

" Cormac Newark, "Ceremony, Celebration, and Spectacle in La Juive«, in: Reading Critics Reading, hg. Roger Parker und Mary Ann Smart, Oxford: Oxford University Press 200I, I 5 5-1 87: I 8 If; ders., Staging Grand Opéra: History and the Imagination in Nineteenth-Century Paris, Ph.D. Oxford University: Oxford University Press 1999, I67.

I2 Vgl. Arnold Jacobshagen, »Analyzing mise-en-scène, Halévy's La Juive at the Salle Le Pelletier «, in: Stage Music and Cultural Transfer: Paris I830-1914, hg. von Mark Everist und Annegret Fauser, Chicago: Chicago University Press 2009, I 76-1 94.

${ }^{13}$ L.-V. Duverger, / Bureau de Commission Théâtrale, rue Rameau, nº. 6. / MISE EN SCENE ET DECORATIONS / DE / LA JUIVE, / Grand Opéra en cinq Actes, Paroles de M. SCRIBE, Musique de M. AUBER [sic!], / REPRESENTE A L'ACADEMIE ROYALE DE MUSIQUE, LE 23 FEVRIER I835. Bibliothèque nationale de France, $4^{\circ}$ Yf. $6 I_{4}$ (4obis). Über Duverger, vgl. Jacobshagen, Staging at the Opéra-Comique, 245 . 


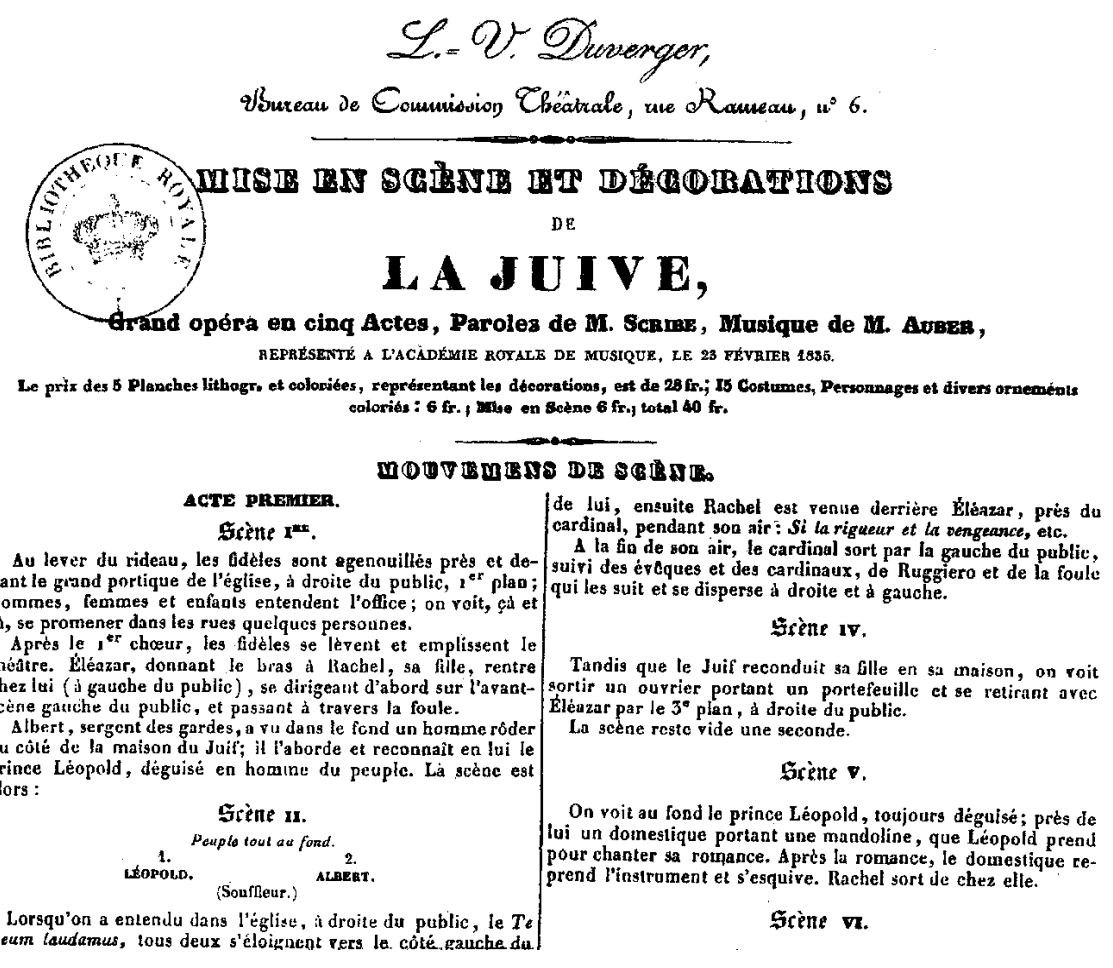

Abb. 2: Louis Duverger, Mise en scène zu La Juive von Auber

Dass die Musik in dieser älteren Quelle fälschlich dem damals weitaus berühmteren Auber zugeschrieben wird und nicht dem Debütanten im Genre der Grand Opéra Halévy, ist nur ein besonders kurioser Hinweis auf die Authentizität des Dokuments. Der Vergleich mit Palianti zeigt, dass es sich um eine andere Inszenierung handelt, und dass die Idee einer werkhaften Konservierung der Originalinszenierung somit weithin eine Fiktion ist.

Auffällig ist, dass bereits Paliantis auf der Titelseite abgebildeter Bühnengrundriss weder mit den Angaben im Libretto, noch mit den bekannten Illustrationen (etwa der berühmten Lithografie von Cicéri) übereinstimmt. ${ }^{14}$ Eléazars Haus steht dort nicht links, wie im und auf den originalen Dekorationen - sondern genau in der Bühnenmitte. Weit schwerer aber wiegt, dass es

${ }_{14}$ Die berühmteste Illustration ist eine kolorierte Lithografie von Cicéri. Vgl. Catherine Join-Diéterle, Les décors de scène de l'Opéra de Paris à l'époque Romantique, Paris: Picard I 988, 37. 
sich nicht mehr nur um einen Leinwandprospekt handelt, sondern bereits um einen dreidimensionalen Bühnenaufbau von erheblicher Tiefe, der mehrere Gassen der Bühne einnimmt. Der von Palianti abgebildete sehr komplex gegliederte Grundriss erfordert aufwendige, begehbare Podestaufbauten, Rampen und Treppen, von denen 1835 natürlich noch keine Rede war. Zudem wird ein gerundetes chassis en panorama als Bühnenhintergrund angegeben, ein Ausstattungsmerkmal, das I835 an der Pariser Opéra ebenso wenig existierte, wie die bei Palianti angegebenen psychologisierenden Beleuchtungseffekte.

Exemplarisch lassen sich die grundsätzlichen Unterschiede beider Inszenierungen am Beispiel des V. Aktes zeigen, der in der grausamen Hinrichtung der Protagonistin in einem Kessel mit kochendem Wasser kulminiert. I 835 zeigte man eine auf Leinwand gemalte und auf eine Holzkonstruktion aufgezogene Hinrichtungsstätte. Dahinter befanden sich zwei Matratzen, auf welche die Primadonna Cornélie Falcon vermutlich allein in der Premierenspielzeit an 46 Vorstellungsabenden gestoßen wurde. Bei Duverger heißt es hierzu:

«Cette cuve est seulement figurée; deux matelas dans ce carré reçoivent l'actrice ou celle de même taille exactement qui la remplacerait; mais il est préférable que l'actrice se prête elle-même à cette action pour plus de vérité et d'intérêt. " ${ }^{\text {Is }}$

Paliantis Beschreibung der entsprechenden Ereignisse aus den I 860er-Jahren liest sich etwas anders. Hier ist von einem enormen Bronzekübel die Rede, der über einem Ofen aus echten Ziegelsteinen platziert ist, unter dem das Feuer bereits lodert. Die Hinrichtung indes wird von zwei Kindern in Henkerkostümen vollzogen, die anstelle der Primadonna eine Puppe in den dampfenden Kessel stoßen:

«Pour l'illusion et la perspective, ce sont deux enfants de douze à treize ans qui, sur la plate-forme, paraissent habillés comme les exécuteurs. C'est aussi une fausse Rachel ou un mannequin que l'on précipite dans la cuve.»

Die Änderung des Bühnenbildes, die Einführung des Rundhorizontes, die außerordentlichen Beleuchtungseffekte und die Ersetzung der besonders unfallgefährdeten Primadonna durch einen wasser- und hitzebeständigen Dummy sind nur einige besonders signifikante Abweichungen zur Premiereninszenierung. Die Tatsache, dass Palianti also keineswegs die originale, sondern eine von dieser stark abweichende Mise en scène $\mathrm{zu}$ La Juive veröffentlichte, zeigt unmissverständlich an, dass die originale keineswegs als sakrosankt, sondern im Gegenteil bereits nach kurzer Zeit als überholt empfunden wurde.

is Duverger, op. cit. [p. 4]. 
Was aber bleibt von den übrigen angeblich originalen Inszenierungen der Grand Opéra, und wie sind diese zu bewerten? Das älteste in diesem Zusammenhang zu betrachtende Werk ist Aubers La Muette de Portici (1828).

\section{INDICATIONS GÉNÉRALES}

ET OBSERVATIONS

POUR

\section{LA MISE EN SCÈNE}

DE

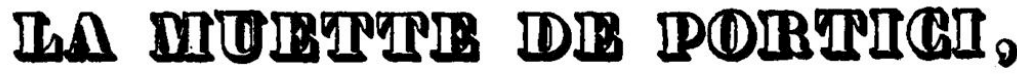 \\ (Grand Gipéra en cinq actes, \\ Parores de mim. SGRIBes ax G. DEhavigne, \\ MUSipUe dx M. AUBER,}

PAR M. SOLOMÉ,

HÉGISSETR GESERAL DE LA NISE EN SCENE DE L'ACADÉMIE GOTALE DE MUSIQUZ.

Abb. 3: Louis-Jacques Solomé, Mise en scène zu La Muette de Portici von Auber

Louis-Jacques Solomés mise en scène ist das bei weitem detaillierteste gedruckte Regiebuch, das wir aus dieser Zeit besitzen. ${ }^{16}$ Ein Hinweis in Franz Grüners I 84 I in Wien erschienenem Buch Die Kunst der Scenik legt zudem die

16 Vgl. Herbert Schneider/Nicole Wild (Hg.), La Muette de Portici. Kritische Ausgabe des Librettos und Dokumentation der ersten Inszenierung, Tübingen: Stauffenburg 1993. 
Vermutung nahe, dass Solomés Inszenierungsanweisungen auch international relativ weit verbreitet waren:

»Es hat auch auf Teutschlands Bühnen vorteilhaft gewirkt, wie uns die Scenerie der Stummen von Portici unwiderlegbar beweiset, welche auf allen Theatern gleichwirkend in Scene gesetzt worden ist, noch nie wurde eine Oper gegeben, deren Scenerie so allgemein als vollkommen gelungen angerühmt worden ist, als die >Stumme<, und dieses rührt daher, weil der Regisseur-General Solome in Paris, seine Composition der Scenerie drucken ließ und dem Werke beilegte. Die teutschen Theater befolgten diese Angabe und die Mehrzahl teutscher Bühnen, welche von dem wahren Rangement keinen Begriff hatten, waren beinahe musterhaft in der Scenen-Setzung oben benannter Oper, und mancher Regisseur gewann dadurch eine Reputation, die er wol aus eigenen Mitteln nie erlangt haben würde. $\ll^{17}$

Solomé blieb jedoch - entgegen der Auffassung der eingangs erwähnten Forscher - eine absolute Ausnahme. Kein zweiter Pariser Opernregisseur dieser Zeit hat selbst ein vergleichbares Regiebuch publiziert. Und alle tatsächlich datierbaren Regiebücher der Grand Opéra aus der Zeit vor dem Prophète bleiben in ihrer Aussagekraft eklatant hinter Solomé zurück. Vor allem handelt es sich - mit Ausnahme von La Muette de Portici - nicht um werkhafte Aufzeichnungen aus dem direkten Umfeld der Produktion, sondern angefangen mit den Publikationen Duvergers seit $1829^{18}$ um summarische Protokolle kommerzieller Theateragenten, die nicht unmittelbar am Aufführungsprozess beteiligt waren.

Solomé war auch noch für die Inszenierung von Rossinis Guillaume Tell ( 8 29) verantwortlich. Elizabeth Bartlet hat im Anhang ihrer kritischen Edition eine wesentlich spätere Brüsseler Quelle, eine handschriftliche Kopie aus der Sammlung von Alexandre Lapissida veröffentlicht und diese Entscheidung ausführlich diskutiert. ${ }^{19}$ Mit Sicherheit nicht als authentisches Dokument der Premiere ist dagegen die von Cohen veröffentlichte Abschrift Paliantis einer weiteren Publikation Duvergers anzusehen, die längst nicht so detailliert ist

17 Franz Grüner, Kunst der Scenik in ästhetischer und ökonomischer Hinsicht, Wien: Mausberger I 84 I, I 35 f.

is Vgl. Marie-Odile Gigou, «Conserver le spectaculaire, ou de l'utilité de la conservation des mises en scène ", in: Le Spectaculaire dans les arts de la scène du romantisme à la Belle Epoque, hg. von Isabelle Moindrot, Olivier Goetz und Sylvie Humbert-Mougin, Paris: CNRS 2006, 47- 2.

19 Vgl. Edizione critica delle opere di Gioachino Rossini, I/39: Guillaume Tell, hg. von M. Elizabeth C. Bartlet, I-IV (Partitura), V (Commento critico), VI (Testi), Pesaro: I992; Guillaume Tell di Gioachino Rossini: fonti iconografiche, hg. von M. Elizabeth C. Bartlet, Pesaro: Fondazione Rossini 1996. 
wie die von Bartlet veröffentlichte Quelle. ${ }^{2 \circ}$ Bei genauerer Betrachtung von Paliantis Kopie fällt auf, dass dieser ursprünglich «opera en trois actes » geschrieben hatte. Später ersetzte er «trois » durch « quatre».

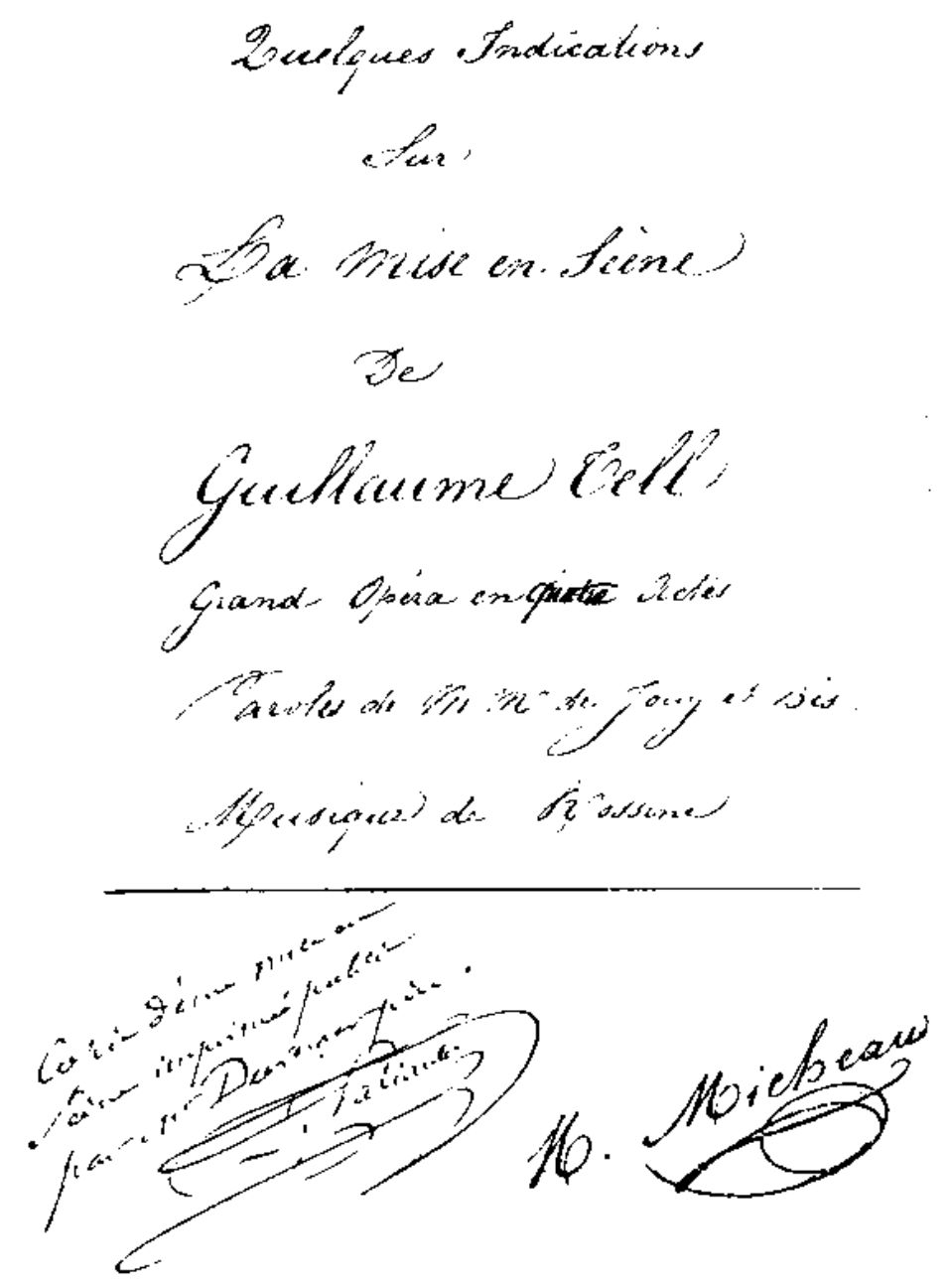

Abb. 4: Louis Palianti, Mise en scène zu Guillaume Tell von Rossini

20 Cohen, The Original Staging Manuals for Twelve Parisian Operatic Premières, 2 I I-229. 
Es ist zu berücksichtigen, dass Guillaume Tell seit I $_{3}$ I in einer dreiaktigen Fassung gespielt wurde, und dass die vieraktige Fassung erst 1856 wiederhergestellt wurde. Vermutlich stammte also Duvergers Quelle aus den dreißiger Jahren, und Palianti veränderte sie in den späten I $850 e r-J a h r e n$. Ein originales Protokoll der Premiere stellen somit Duvergers und Paliantis Aufzeichnungen keineswegs dar.

Als Meyerbeers Robert le Diable i83 I erschien, war Solomé soeben von keinem anderen als Louis Véron entlassen worden - und zwar aus dem bemerkenswerten Grund, dass ausgerechnet der für seine Inszenierungsvorlieben bekannte neue Operndirektor den damals berühmtesten Pariser Regisseur für überflüssig hielt. Wie John Drysdale in seiner Dissertation über die Finanzen der Académie Royale de Musique gezeigt hat, sparte der directeur-entrepreneur Véron, wo er nur konnte, nach Möglichkeit auch an der Inszenierung (was bei der Würdigung von Vérons Leistungen gerade auf diesem Gebiet gerne übersehen wird). ${ }^{21}$ Und bekanntlich wurde gerade die Inszenierung von Robert le Diable nicht nur für ihre außergewöhnlichen Attraktionen, sondern auch für eine Serie gravierender Pannen und Unfälle bekannt. In ihrer Dissertation über die Inszenierung von Robert le Diable hat Rebecca Susan Wilberg die Kopie eines Brüsseler Regiebuches aus dem späten I9. Jahrhundert veröffentlicht - und zwar sowohl als Faksimile wie auch als Transkription - wohl im Vertrauen in die Richtigkeit von Cohens Hypothese. ${ }^{22}$ Der Vergleich dieser Quelle mit einer weiteren Palianti-Kopie nach Duverger zeigt deutliche Unterschiede bereits in dem auf der Titelseite gezeigten ersten Bühnenbild. Auffallend sind auch die Unterschiede im Grundriss des zweiten Bildes des III. Aktes auf. Der Brüsseler Quelle zufolge war der Kreuzgang des Klosters zudem bereits eine Ruine, während die berühmte Pariser Lithografie aus Cicéris Atelier ein verlassenes, aber architektonisch intaktes Bauwerk zeigt. Das von Wilberg transkribierte Regieprotokoll ist wesentlich ausführlicher als dasjenige Duvergers, aber es dürfte sich kaum um eine zuverlässige Dokumentation der Pariser Uraufführung handeln. Es zeigt sich zugleich, dass auch Paliantis wiederum von Cohen als angeblich authentisches Dokument von I83 I veröffentlichte Kopie einer Duverger-Quelle keineswegs als »originales Inszenierungsdokument « angesehen werden kann. So begegnet beispielsweise in keiner der von Wilberg und Cohen veröffentlichten Inszenierungen die Rolle des Alberti, die erst 1832 gestrichen (bzw. teilweise durch einen anonymen

${ }^{21}$ John Drysdale, Louis Véron and the Finances of the Académie Royale de Musique, Frankfurt/Main: Peter Lang 2003 (Perspektiven der Opernforschung 9), 78.

${ }^{22}$ Rebecca Susan Wilberg, The "mise en scène" at the Paris Opéra, Salle Le Peletier (I 82 I-I 873), Ph.D. Diss. Brigham Young University 1990. 
«chapelin de Robert» ersetzt) wurde. In der Premierenfassung tritt Alberti freilich bereits in der ersten Szene auf, und so würden wir erwarten, ihn auch in der originalen Mise en scène erwähnt zu finden. Ein solches authentisches Inszenierungsprotokoll zur Premiere von Robert le Diable ist bislang jedoch leider noch nicht aufgetaucht.

Für Aubers Gustave III (1833) - um in der Chronologie der Grand Opéra fortzufahren - hat Cohen eine weitere Kopie nach Duverger faksimiliert und angegeben, es handele sich um Paliantis Handschrift. Dies ist zweifellos nicht der Fall, wie ein Vergleich mit den bereits diskutierten, wesentlich eleganter geschriebenen Palianti-Manuskripten unzweideutig zeigt. Zudem ist die originale Duverger-Publikation, die zwar nicht in der Bibliothèque der Association de la Régie théâtrale, wohl aber in der Bibliothèque Nationale ${ }^{23} \mathrm{zu}$ finden ist, einer späteren handschriftlichen Kopie als Quelle deutlich vorzuziehen. ${ }^{24}$

Wie im Falle von La Juive sowie seiner übrigen Regiepublikationen benötigte Duverger lediglich zwei doppelseitig bedruckte Foliobögen, um die Inszenierung von Gustave III zu transkribieren, während die großzügige Handschrift in Cohens Edition 44 Seiten füllt. Gleichwohl ist auch diese Kopie nicht mit der gedruckten Vorlage identisch, sondern enthält deutliche Hinweise auf spätere Konventionen. Hier ist die Dekorationsbeschreibung in den Haupttext integriert und nicht als separate Liste an dessen Ende gestellt, wie es noch bei Solomé und Duverger üblich war. Das Manuskript weist darüber hinaus keine Kürzungsvorschläge der Ballettnummern auf, wie wir sie bei Duverger finden. Der wichtigste Unterschied betrifft jedoch die Kostüme, die im späteren Dokument ganz anders beschrieben werden. Dies deutet darauf hin, dass diese Kopie mit Blick auf eine bestimmte Aufführung angefertigt wurde. Nach Gustave und La Juive bildet Meyerbeers Les Huguenots das vorerst letzte prominente Beispiel. Auch zu diesem Werk hat Cohen eine handschriftliche Palianti-Kopie nach Duverger veröffentlicht, die bereits quellenimmanent zahlreiche Veränderungen dokumentiert, darunter überwiegend Kürzungen, aber auch solche Änderungen, die allein die Regie und nicht die Musik betreffen. ${ }^{25}$

23 Bibliothèque nationale de France, $4^{\circ}$ Yf. I I 4 (33).

24 Cohen, The Original Staging Manuals for Ten Parisian Operatic Premières, I3-55.

25 Cohen, The Original Staging Manuals for Ten Parisian Operatic Premières, I33-172. 


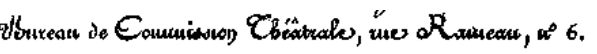

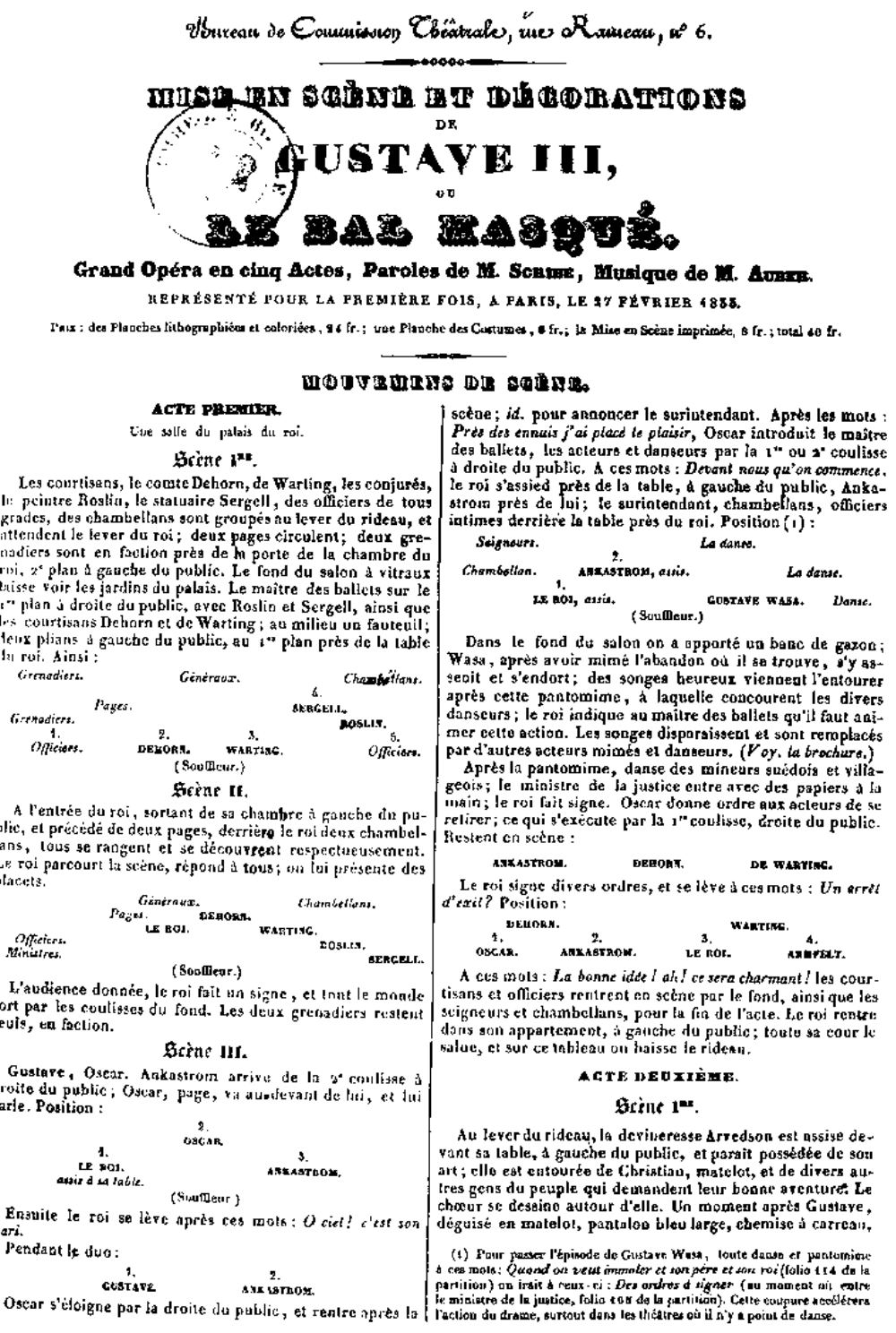

Abb. 5: Louis Duverger, Mise en scène zu Gustave III von Auber 
Es sei nur daran erinnert, dass Werke wie Gustave III, La Juive oder Les Huguenots bei auswärtigen Aufführungen schon aus Zensurgründen kaum je in der »originalen Werkgestalt« geschweige denn in der »originalen Inszenierung" gespielt werden konnten. Im deutschsprachigen Raum beispielsweise, dem bei weitem wichtigsten auswärtigen Markt für französische Opernproduktionen, wurde Gustave III zunächst unter dem Titel Die Ballnacht gespielt, mit einer Handlung im I6. Jahrhundert und einem gewissen »Herzog Olaf« in der Titelrolle. ${ }^{26}$ Dass für solche stark veränderten Werkfassungen - die für die europäische Verbreitung der Grand Opéra in dieser Phase nicht etwa die Ausnahme, sondern die Regel waren - die sklavische Befolgung eines Pariser Inszenierungsdokuments ohnehin nicht in Frage kam, dürfte sich eigentlich von selbst verstehen. Ganz abgesehen von der fraglichen Authentizität aller Inszenierungsprotokolle der frühen Grand Opéra - mit Ausnahme von La Muette de Portici - sind diese überwiegend sehr summarisch angelegt und beschränken sich auf einfache Personenarrangements sowie Dekorations- und Kostümbeschreibungen. Ein erheblicher Teil dieser Informationen stammt dabei ohnehin aus den Regieanweisungen des Librettos. Als Fazit der bisherigen Beobachtungen ist also die bisher gehegte Vorstellung einer gleichsam »werkhaften«, weil schriftlich fixierten Inszenierung zumindest für die Grand Opéra der dreißiger und vierziger Jahre in ihrer historischen Bedeutung unbedingt zu relativieren: nämlich als bloße Rückwärtsprojektion moderner Werkkonzeptionen, aber gewiss nicht als Ausdruck der zeitgenössischen Bühnenrealität und Aufführungspraxis.

Unter diesen den bisherigen Forschungsstand relativierenden Voraussetzungen erhalten die Inszenierungsanweisungen Paliantis zu Meyerbeers Le Prophète einen etwas anderen Stellenwert. Die Erstveröffentlichung der Inszenierungshinweise erfolgte als Supplement in der Revue et Gazette des Théâtres im November I849, also etwa ein halbes Jahr nach der Premiere (16. April r 849). Der Autor Louis Palianti, I8 10 in Spanien geboren, kam als Kind mit seiner Familie nach Frankreich und schlug sich später als zweiter Bassist an den Provinzbühnen von Nantes, Marseille und Dijon durch, ehe er I 836 an der Opéra-Comique engagiert wurde. Dort hatte er fast vierzig Jahre lang zugleich die Funktionen des Sous-Regisseurs und die eines Nebendarstellers im Bassfach inne. 1838 begann er mit der regelmäßigen Publikation von Regieprotokollen zu Werken der Opéra-Comique. ${ }^{27}$ Diese Inszenierungen

26 Vgl. Arne Langer, Der Regisseur und die Aufzeichnungspraxis in der Opernregie im I9. Jabrbundert, Frankfurt am Main: Peter Lang 1997 (Perspektiven der Opernforschung 4), 316.

${ }_{27}$ Vgl. die Liste der Publikationen Louis Paliantis bei Arne Langer, ibid. 349-356. 
fasste er später in Bände und Serien zusammen. I84I veröffentlichte Palianti erstmals eine Inszenierung der Opéra, nämlich Donizettis La Favorite. Es folgten in sehr unregelmäßigen Abständen noch fünf weitere Hefte zu Werken der Opéra, jeweils zwischen vier und maximal sechzehn Seiten lang: Nach Les Martyrs und Dom Sébastian desselben Komponisten sowie La Reine de Chypre von Halévy und L'Ame en peine von Flotow war Le Prophète überhaupt erst die sechste Inszenierung einer Opéra, die Palianti in den Druck gab. Mit diesen nur summarisch behandelten Vorgängerwerken - anders als an der Opéra-Comique war Palianti an den Produktionen der Académie Royale de Musique persönlich nicht beteiligt - ist das 32 Druckseiten umfassende Regiebuch zu Le Prophète hinsichtlich der Differenziertheit der szenischen Angaben kaum zu vergleichen.

Bereits an den Bühnenbildbeschreibungen zu Le Prophète ist zu erkennen, dass diese nicht mehr - wie in den früheren Protokollen Paliantis zu Produktionen der Opéra - das bereits im Libretto angegebene Dekor in seinen summarischen Beschreibungen wiederholen, sondern unter Zuhilfenahme des Bühnengrundrisses wesentlich konkretisieren. Hatte Palianti noch bei Donizettis Les Martyrs (I84I) auf Bühnengrundrisse gänzlich verzichtet und bei Dom Sébastian (I 843) diese nur vereinfacht dargestellt, erlauben die Grundrisszeichnungen und ihre Erläuterungen im Fall von Le Prophète eine recht genaue Rekonstruktion. Im Libretto Eugène Scribes heißt es zu den Dekorationen des I. Aktes lediglich:

«Le théâtre représente les campagnes de la Hollande aux environs de Dordrecht. Au fond on apperçoit la Meuse; à droite un château-fort avec pont-levis et tourelles; à gauche, fermes et moulins dependant du château. Du même côté, sur le premier plan, des sacs de blé, des tables rustiques, des bancs, etc. etc. ${ }^{28}$

Die szenische Beschreibung Paliantis im Regiebuch geht zunächst gar nicht von der verbalen Umschreibung, sondern von der bildlichen Darstellung, d.h. hier konkret vom Bühnengrundriss aus. Von Scribes Szenenangabe im Libretto ist nur die Zusammenfassung geblieben: «Les campagnes de la Hollande aux environs de Dordrecht. »

${ }_{28}$ Fabien Guilloux, Giacomo Meyerbeer. Le Prophète. Livret, Sy. 9045, München: Ricordi 2007, 99. 


\section{Gupplément à la Revie et Gazette des Théâtres.}

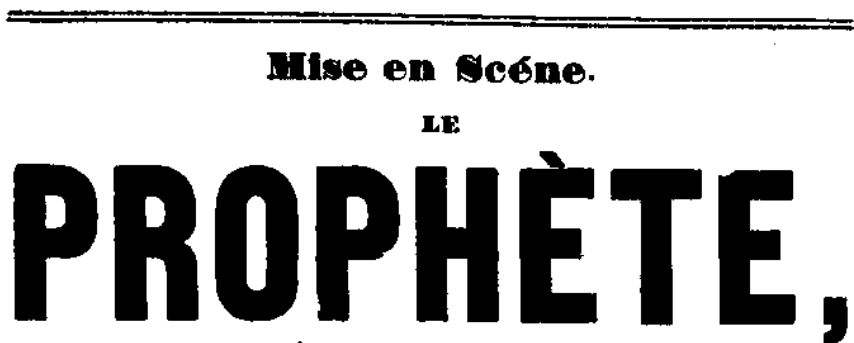

OPERA EN CINQ ACTES (1),

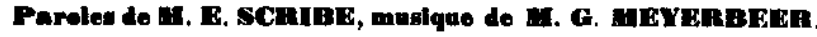

Représenté pour la première tois, à Paris, gur le théátre de l'Opéra, le 16 avril 1849 .

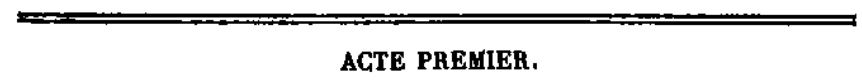

Les campegnes de la Hollande aux environs de Dordrecht.

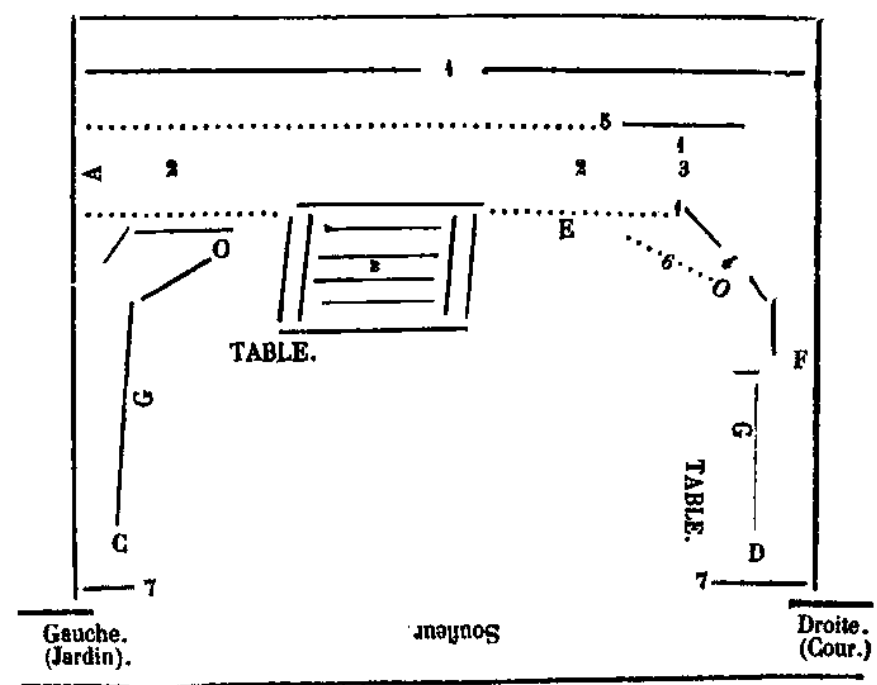

(1) Pertilion, parties d'orchestre, et morcesux detachls pour le piazo; Peris, ches Mi. BRANDUS et comp., rue Rlchelieu, B7, ancien 87.

Abb. 6: Louis Palianti, Mise en scène zu Le Prophète, Akt I, Grundriss 
Die verbale Beschreibung liefert sodann im Wesentlichen die technischen Informationen zur exakten Umsetzung der in der Grundrisszeichnung festgelegten Dispositionen:

\begin{abstract}
"Rideau de fond tombant sur le quatrième plan. Ce rideau représente une suite de moulins à vent sur les bords de la Meuse. - Celui qui, au premier plan, occupe presque toute la hauteur du Rideau, se trouve vers le côté jardin, presque en face des marches B.2. Terrain praticable élevé à deux mètres environ au dessus du niveau du théâtre. - De la coulisse (côté jardin) on monte sur ce terrain par un escalier A, non vu du public. - A la droite 3 , se trouve l'entrée principale, avec pont-levis, du château-fort d'Oberthal. - Les murs 4, occupant une partie du côté cour. - Les tourelles 5 servent à la perspective du Rideau de fond. - Du terrain 2, on descend sur le théâtre par un escalier de pierre B. Quelques herbes marécegeuses poussent au pied du terrain 2. - A l'endroit où, dans le tableau ci-dessus est un $\mathrm{O}$, se trouve un arbre au tronc très-haut, mais peu large; le haut du feuillage de cet arbre se joint presque à celui d'un arbre côté jardin. (Sujet de la frise tombant à cet aplomb.) Aux ailes cour et jardin, châssis représentant un des côtés des fermes dépendant du château. - 7. Draperie. - C. Entrée et sortie côté jardin. - D. Entrée et sortie côté cour. -Eclairez magnifiquement cette décoration, qui doit offrir un aspect riant. - Des tables rustiques, des bancs et des sièges de bois sont placés aux endroits indiqués dans le tableau. »
\end{abstract}

Neben der genauen Anordnung der einzelnen Bildelemente enthält diese Beschreibung vor allem exakte Höhenangaben, also eine Präzisierung der dritten, im Grundriss nicht darstellbaren Dimension. Da die Inszenierungsanweisungen auf unterschiedliche Bühnenmaße übertragbar sein mussten, stehen hier relative Höhenangaben (z.B. «presque toute la hauteur du Rideau ») neben absoluten Maßen (z.B. «Terrain praticable élevé à deux mètres environ au dessus du niveau du théâtre »).

Die Bedeutung einer exakten Bestimmung der Bühnendimensionen und der Verhältnisse der einzelnen Bühnenbildelemente zueinander zeigt sich vor allem bei den Verwandlungen, die sowohl zwischen dem ersten und II. Akt als auch innerhalb des dritten und IV. Aktes bei offener Szene erfolgen. Logistisch besonders komplex ist die Verwandlung des IV. Aktes, der ja nicht nur das für Le Prophète zentrale Krönungstableau im Dom zu Münster umfasst, sondern dem zuvor das erste Bild auf einem öffentlichen Platz dieser Stadt vorangeht. Eugène Scribes Libretto ist nicht zu entnehmen, dass diese beiden sehr unterschiedlichen und sehr komplexen Dekorationen gleichzeitig (nur durch einen Vorhang getrennt) auf der selben Bühne Platz finden müssen. Über das erste Bild heißt es im Libretto:

«Le théâtre représente une place publique de la ville de Munster. A droite la porte de l'hôtel de ville de Munster; plusieurs marches y conduisent. Plusieurs rues aboutissent à 
Oper als SZenischer TeXt: Paliantis InSZEnIERUngsanweisungen Zu LE PROPHÈTE

la place publique. Au lever du rideau plusieurs bourgeois, portant des sacs d'argent ou des vases précieux, montent les marches de l'hôtel de ville; d'autres descendent les mains vides. Plusieurs arrivent par les différentes rues, s'avancent au bord du théâtre et forment des groupes. Il regardent autour d'eux avec inquiétude et se parlent à voix basse. »

Wie erst aus Paliantis Angaben ersichtlich, nehmen diese Gebäude und Straßen lediglich die Vorderbühne ein:

«Une place publique de la ville de Munster. - Selon la profondeur des théâtres Rideau tombant à l'aplomb du troisième, quatrième ou cinquième plan, juste au pied des marches de la décoration qui se trouve derrière. » 


\section{ACTE QUATRIEME.}

Utre place publique de la ville de Munster. - Selon la profondeur des théstres Ridesu tombant à Paplomb du troisieme, quatrieme ou cinquiène plan, juste au pied des marclies de la décoration qui so trouve derrière. - Peint sur le rideau, face au public, vers la cour, est l'entrée d'ua pont (A) tournant censé vers le côté jardin, et soutissant de ce côté, droit à une rue censee ou lointain cour (entrée B). - Devant ce rideau, à quelques centimètres de distance, est un chássis représentant le parapet du pont. Ce chassis, dispose sur un trapillon, disparail dans le dessous at changement; tl occupe toul le cóté jardin jusgu'à la perpective de l'entrée du pont, où, sur un plédestal, se trouve une slatue (C) - - Le parapet et la statue du cóté opposé sont peints sur le ridesu. - Une grosse pierre est au pied du porapet (D). C'est sur cette pierre que Bides, accablée de fatigue, vient s'asseoir. - Châssis de place publique (archilecture gothique) sur les premiers plans cour et jarditt. - Le chasssis de lointain, jardin (F) représente l'entrée d'une vieille église abandinnnée. - Le châssis gui fait face (G) represente l'entrée de l'Elôtel-do-Ville. - E. A., entrées cóté jardiv. - B. I., entres côté cour.

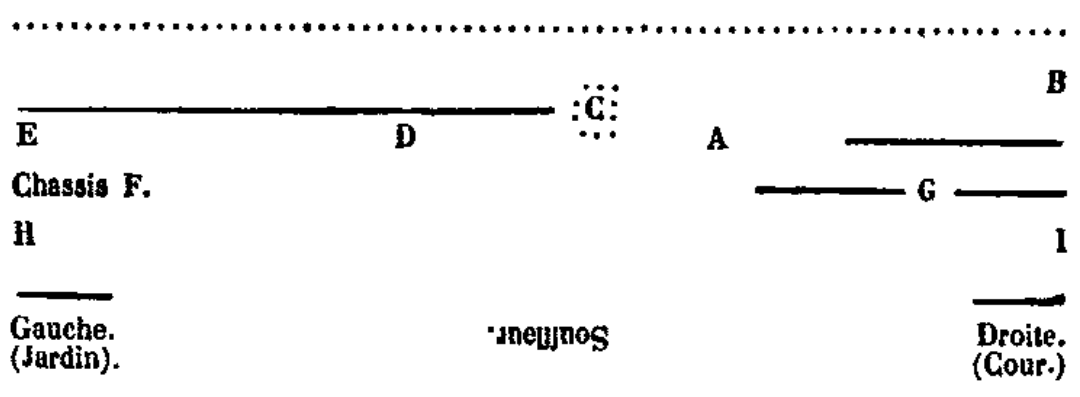

Au lever de rideau, plusieurs groupes de bourgeois, dhommes du peuple et de guerriers, causant bas entre eux, occupent la scene: (Ténors au jardin, q" ténors gu milieu, basses à la cour.) Courbons notre tete, craignons les mechass. Voict la tempete, voict les méchans. - Pendant ce chour, plusieurs bourgeois portant des sacs d'argent ou des vases précieux, entrent à l'Hótel-de-Ville; d'autres en sortent trislement, les mains vides, et se joignent sux groupes qui sont en scèue. - Tous regardent autour d'eux avec inquiétude. - De nouveaux bourgeois (coryphèes) débouc hent par les issues B. E., descendent vivement en scéne, el font comprendre qu'une patrouille s'avance. Tous chantent alors a haute voix: Vive le prophètel Yivent ses soldats \ Puis, redescendant plus en scène, ils disent à voix basse : 1 bas le Prophete $1 \dot{a}$ bes ses soldats!

Abb. 7: Louis Palianti, Mise en scène zu Le Prophète, Akt IV

Bei der hinter dem «Rideau tombant à l'aplomb du troisième, quatrième ou cinquième plan » befindlichen Dekoration handelt es sich selbstverständlich um jene des zweiten Bildes, über die es im Libretto heißt:

«Le théâtre change et représente la cathédrale de Munster. Une partie du cortège est censée déjà entrée, l'autre moitié continue à défiler; au fond de l'église des trabans de la 
garde du prophète forment la haie. Marches des grands électeurs portant l'un la couronne, l'autre le sceptre, l'autre la main de la justice, celui-ci le sceau de l'Etat, et d'autres les ornements impériaux. Jean paraît après eux, la tête nue et habillé en blanc. Il traverse la nef principale et se rend dans le chœur au maître-autel qui est dans le fond à droite et qu'on ne voit pas. Le peuple qui est sur le devant du théâtre veut se précipiter sur ses pas. Il est repoussée par les trabans dans les chapelles latérales. »

Dass sich der Bühnenbildwechsel vom ersten zum zweiten Bild des IV. Aktes bei offener Szene, als "changement à vue» vollzieht, setzt voraus, dass die Kulissen des ersten Bildes - wie bei Palianti angegeben - aus Leinwandprospekten bestehen, die während der Verwandlung bei gleichzeitigem Heben des hinteren Vorhangs innerhalb von Sekunden beiseite gezogen werden. Die einzige Ausnahme bildet ein Brückengeländer, das im Moment der Verwandlung in einer Versenkung im Boden verschwindet («Devant ce rideau, à quelques centimètres de distance, est un châssis représentant le parapet du pont. Ce châssis, disposé sur un trapillon, disparaît dans le dessous au changement. ») Die hierdurch freigegebenen vorderen Bühnensegmente dienen sodann für den Aufzug der Menschenmassen in der Krönungsszene. 


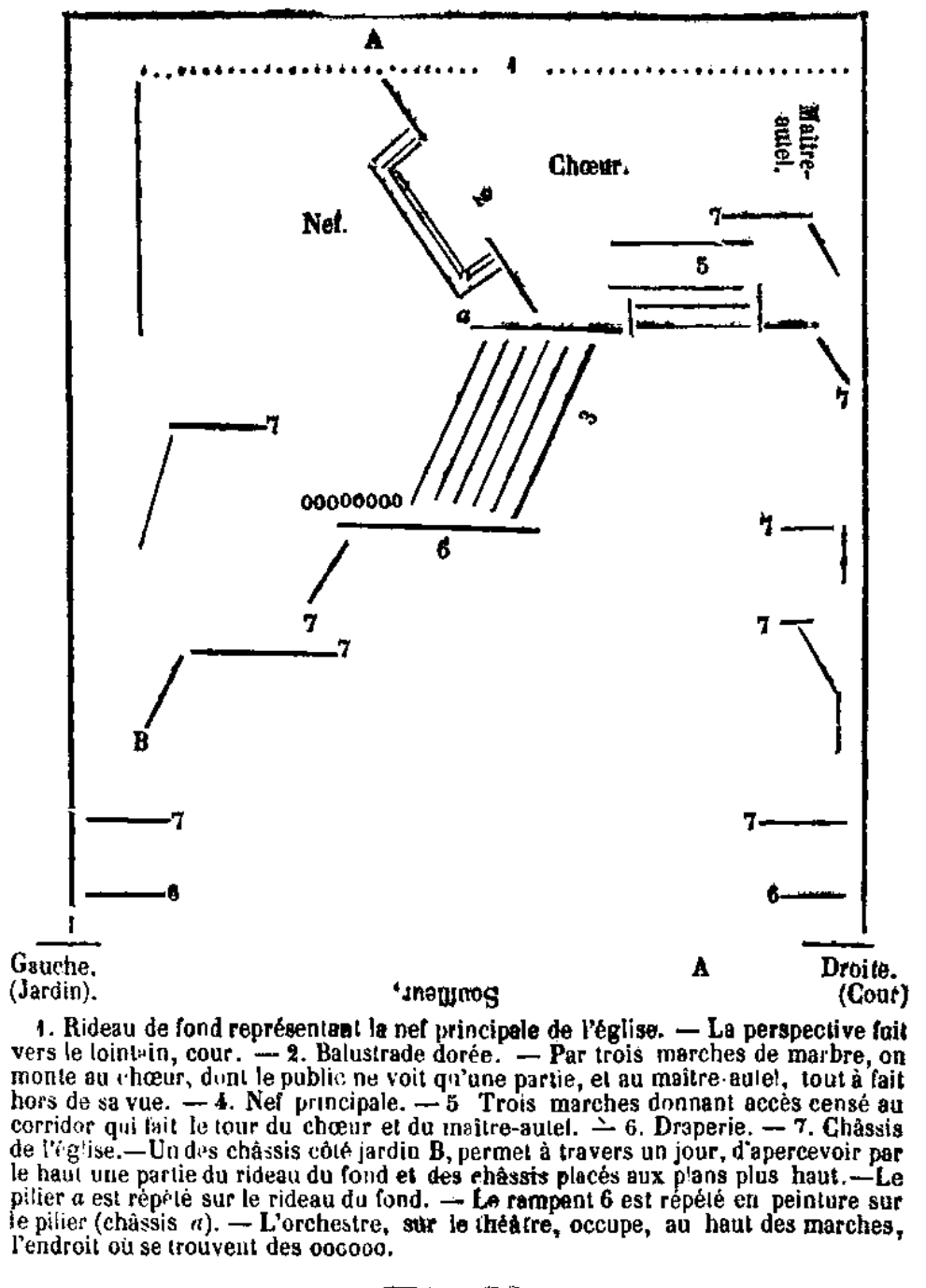

Des Hommes, des Femmes et des Enfans du peuple occupent lo théatre, et descendent en scène.-Six Soldals (T'abans du Prophèie), descendent eu mêrne temps les marches du fond, laissent deux des leurs en faction au pied de l'escatier, puis ils repoussent le Peuple vers les chapelles latérales, et veillent à contenir la foule.

Abb. 8: Louis Palianti, Mise en scène zu Le Prophète, Akt IV 
MABChE DU SACRE.

Placez la musique miliaire sur le theiglre, en vue du public, sur le sol de la nef, à l'endroit indiqué par des 0000 dans le tableau ci-dessns. - La marche se renadiat au chueur el all natle-aulel, 1raverse processionnellement au haul des marches, la nef principj 4, de jardin à cour, dans l'orure suivant:

1. Vingt Soluals, deux yar deux, précédés d'un Chel. - 2' Donze Enfans de chour. - $3^{\circ}$ Quatre Moines en rotues vinteiles, toques carrées, surplis de guipure, pél rines à capuchon, elc. - $4^{\circ}$ Douze autres Evilians de chour portant des encensoirs. Ils se rangent en vue du public, de chaque côte des marches $2_{\text {, qui con- }}$ duisent au maitre-aulel. - - $\mathbf{z}^{*}$ Un Hérault d'armes portant la bannière du Prophète. $-6^{\circ}$ Huit Paurs. $-7^{\circ}$ Douze grands Elecleurs portant sur des coussins de velours, lun la couronne, lautro le sceptre, l'autre la main de justice, ct-lui-ci le sceau de l'Etat, et d'auires les ornenens impériaux, - I es Enfans de chour tncensent.-La marche continue. - $8^{\circ}$ Magnifique dais, porié par deux Hé1auts d'armes, et sous lequel se trouve le Prophètu la lête nue, el habillé en blanc. Près do luj, en dehors du dais, marcheot Jonas, Mathisen et Zacharı. - Le cortẹge s'arrête un instant pendart que les Eifans de chour encensent. Ces derniers suivent la marche.- go Trois Grands Dignitcires sunvis de leurs Pages. $-10^{\circ}$ Douze Hogistrats aux grands manteaux de d aj d'or doub és d'hermine. - ifo Trois autres grands Dirniraires de l'armée, suivis de leurs Pages. -120 Un Héraut d'armes portant une bannière. $-13^{\circ}$ Trois autres Dignitaires de l'armee suivis de leurs Pages. - 160 Douze clairons. Les insll umens surt ornès de tabil $\mathrm{s}$ armuiriés. - $15^{\circ}$ Des Soldats finissent la marche. Pendant cele marcbe, des Hallebardiers et des Trabans du Prophèle ont gardé le haut le bas des mrrches 2 , el les quatre coins de la nef, qui précede les marches el la balustrade d'or $z$, qui donne entrée dans le choeur, dépendant du, mailre-autel. Vers la fin de la marche, Fidès est entree sur la scène par un des seconds pláns de gauche, et se mêle au Peuple. - Pendant le roulement de tambour qui s'exécule au haut des marches, a la place qu'occupaient les Musiciens, les Soldats et les Hallebardiers qui sont du hout, meltent un genoux en terre. Ceux qui sont en scène, en tont autant sur les marches. Le Peuple, divisé en plusieurs groupes, garnit au fond toute la largeur du thestre et les marches. - Pour la perspecije, placez des Enfans agenovilles tout y fajt au fond. côté cuur. Tous les personinges vus du public en ce moment, sont agenouiliés, tournent le dos au public, et portent leurs regards vers le maitre-aulel. - Fidès seule, presque tace au public, est agenouillé regards vers le maitre-autel.

Le pomine salvum fac regen se chante dans la coulisse cỏlé cour censé au maitre - autel. - Les or rues se font entendse. - Les pesonnages vus du pu-

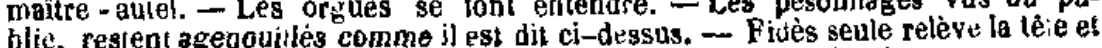
s'écrie: Oue Dier sauce le roi Prophète, disent-ils?.. Grand Dieu exauces ma stère et gu'errant misérable et proscrit, il soit mitudit. S'anisnanl pas degtés, priere, el qu'errant, mise o ! ma fille, d Judith nouvelle, etc., puis elle se lève sur les derniets mots: Le Stigneur conduira ton bras.

Abb. 9: Louis Palianti, Mise en scène zu Le Prophète, Akt IV, Bild 2, Cortège

Die minutiöse Beschreibung des Cortège in der Krönungsszene knüpft an ältere Beispiele der Grand Opéra an, etwa an den Einzug des Kaisers und der weltlichen und geistlichen Würdenträger in Halévys La Juive. In dieser Auflistung werden weit über hundert Personen individuell bzw. als Angehörige kleiner Gruppen beschrieben, die im Cortège nacheinander erscheinen und 
denen eine nicht spezifizierte Anzahl von Soldaten folgt. Die Gesamtzahl der auf der Bühne versammelten Personen ergibt sich aus dem Cortège der Statisten und Solisten (darunter Jean und die drei Wiedertäufer) sowie dem bereits im Moment der Verwandlung auf der Bühne (d.h. in der Kirche) befindlichen Chor des Volkes ( Des Hommes, des Femmes et des Enfans du peuple occupent le théâtre, et descendent en scène. »).

Innerhalb des III. Aktes gibt es sogar zwei Verwandlungen bei offener Bühne: Das ursprüngliche Bild, das das Lager der Wiedertäufer und den vereisten See repräsentiert, verwandelt sich bereits nach der ersten Szene. Gezeigt wird sodann das Innere des Zeltes von Zacharias. Nach der vierten Szene wird erneut auf das anfängliche Dekor zurückgegriffen. Diese Verwandlungen sind insofern von Interesse, als sie - bei kaum veränderter Konstellation der beteiligten Hauptfiguren - einen dem Wechsel der Einstellungsgrößen im Film durchaus vergleichbaren Übergang von der Panoramaaufnahme der Landschaft in den Innenraum des Zeltes und wieder zurück vollziehen.

Das erste Panoramabild endet mit dem Ballett der Schlittschuhläufer, die während des abschließenden Galopp den See entvölkern:

«A l'exception des danseurs, le personnel en scène devient graduellement moins nombreux. - Tableau très-animé. - Vers la fin du galop, il ne passe presque plus personne sur le lac glacé. » 
ACTE TROISIËME.

Le camp des Angapatistes dans une forêt de Westphalie.-En face du spectateur, Lu etang des qui s'etend à l'horizon. - Au loin, dans les brouillards et les nuages, on apercoit la silhouetle de la ville de Munster.-Etfet de neige.

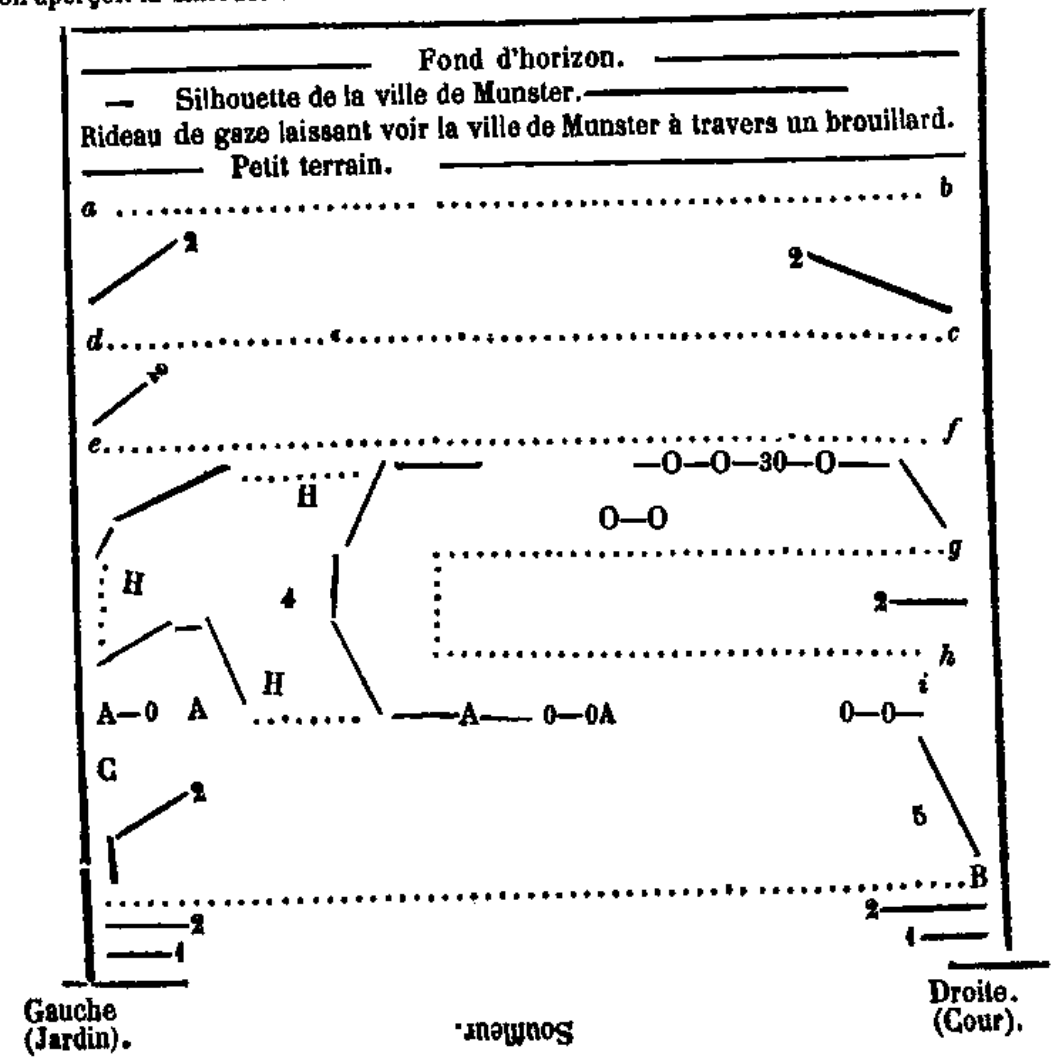

Abb. Io: Louis Palianti, Mise en scène zu Le Prophète, Akt III

Paliantis Szenenanweisungen zum Ballett der Schlittschuhläufer sind auch wegen ihrer präzisen Berücksichtigung der perspektivischen Verhältnisse von Interesse. ${ }^{29}$ Die Schlittschuhläufer wurden gemäß ihrer Körpergröße geordnet

29 Hierzu siehe auch Arnold Jacobshagen, »Chœur dansé und Chœur en action. Zur szenischen Realisierung bewegter Chöre in der französischen Oper«, in: Bewegung im Blick. Beiträge zu einer theaterwissenschaftlichen Bewegungsforschung (Documenta choreologica-Studienbibliothek zur Geschichte der Tanzkunst, Tanzarchiv Leipzig), hg. von Claudia Jeschke und Hans-Peter Bayerdörfer, Berlin: Vorwerk 2000, 29I-306. 
und bildeten parallel zur Bühnenrampe gestaffelte Reihen, so dass sich eine perfekte perspektivische Illusion ergab:

«Pour la perspective, faites traverser des enfans de a à b. Des jeunes gens d'une moyenne taille de c à d, et de e à f, puis en dernier lieu de grandes personnes de gà h, qui entrent en scène par la droite i. »

Von Interesse ist auch die Nennung des Herstellers der anstelle der Schlittschuhe verwendeten Rollschuhe: «Les patins à roulettes sont de l'invention de M. Legrand, 8, rue des Jardins, à Chaillot. » Die tatsächliche Herausforderung dieser Erfindung bestand darin, Rollschuhe zu entwickeln, die selbst bei gleichzeitiger Verwendung durch eine große Personenzahl auf dem Bühnenboden des Theaters möglichst geräuschlos einsetzbar waren, denn andernfalls wäre die Illusion des vereisten Sees vollkommen zerstört worden. Außergewöhnlicher noch war freilich die technische Erfindung der im selben Akt zum Einsatz kommenden Prophetensonne, die in der Literatur bereits eingehend erörtert wurde. ${ }^{30}$ Auch hier gibt Palianti die genaue Herstelleradresse an: «A l'Opéra, le lever du soleil s'exécute au moyen de l'appareil électrique de M. Lormier, I3, rue du Delta projetée, à Paris. » Lormier war jedoch nicht der Erfinder, sondern lediglich der Produzent der Pariser Prophetensonne, der Jules Duboscs Realisierung des Sonnenaufgangs mit Hilfe einer von dem Physiker Léon Foucault entwickelten Kohlen-Lichtbogenlampe vertrieb.

Aufschlussreich für eine Bewertung des unmittelbaren Anteils des Komponisten an der Inszenierung sind die zahlreichen sehr präzisen Angaben zur Koordination von Szene und Musik. Ein erstes Beispiel hierfür findet sich bereits zu Beginn der ersten Szene:

«Au lever du rideau, quelques Meuniers, couchés à la face, près des châssis de gauche, se reposent ou dorment sur des sacs de farine. - Un Pâtre, assis, sur le haut des marches B, se lève et donne l'éveil en jouant de la cornemuse. Un autre Pâtre (censé dans la coulisse) lui répond de loin. - Le Pâtre renouvelle ce jeu à plusieurs reprises sur le haut du terrain 2. - Immédiatement après les échos, dès l'attaque de la ritournelle en sol majeur, des Paysannes entrent par la droite D. »

Während diese szenischen Anweisungen im Probenstadium auch ohne weiteres allein mündlich kommuniziert werden könnten, setzen kurz darauf in der Pastorale des II. Aktes die Zuordnungen zwischen Musik und Aktion eine schriftliche Fixierung voraus:

30 Vgl. Matthias Brzoska, «>Wirkung mit Ursache<. Idée esthétique et apparence du spectaculaire dans l'œuvre de Meyerbeer ", in: Le Spectaculaire dans les arts de la scène du romantisme à la Belle Epoque, hg. von Isabelle Moindrot, Olivier Goetz und Sylvie Humbert-Mougin, Paris : CNRS 2006, 84-93. 
«Pendant les quelques mesures qui précèdent la pastorale, Zacharie remonte la scène pour s'assurer si personne ne vient, puis il redescend occuper le numéro 2.

Mathiesen. - Zacharie. - Jean. - Jonas.

A l'ensemble allegro 2/4 qui suit le second couplet de la pastorale, les trois anabaptistes remontent le théâtre en disant à Jean : Ah ! viens, suis nos pas, etc., etc. - Ce dernier fuit, au contraire, vers l'avant-scène de gauche. - Les quatre dernières mesures de l'ensemble Et bientôt tu règneras, se chantent presque sur le seuil de la porte du fond 2. Immédiatement après la dernière note chantée, les trois anabaptistes s'éloignent dans la campagne vers le côté Cour. »

Die fortschreitende Präzisierung in der Zuordnung von Aktionen zu konkreten Taktangaben, wie sie sich hier und an zahlreichen anderen Stellen zeigen, sind ein Indiz dafür, dass die Aufzeichnungsform im Livret de mise en scène allmählich unangemessen wurde. Tatsächlich war in der Bühnenpraxis der Übergang zum »durchschossenen « (d.h. mit leeren Zwischenseiten für szenische Einträge versehenen) Klavierauszug unvermeidlich, dessen Durchsetzung im späteren I9. Jahrhundert für das allmähliche Schwinden der Livrets de mise en scène zu dieser Zeit mitverantwortlich sein dürfte.

Mit Blick auf das Verhältnis zwischen Bühnengeschehen und Musik sei abschließend die eingangs erwähnte These erörtert, inwieweit Meyerbeer (als mutmaßlich erster Opernkomponist) mit der Inszenierungsdokumentation des Prophète eine umfassende Kontrolle über die szenische Realisierung seines Werkes auch für alle späteren Inszenierungen und Aufführungen andernorts sicherstellen wollte. Hierin zeigt sich ein deutlicher Unterschied zu den zunächst betrachteten, lediglich summarisch dokumentierten Grands Opéras, deren Inszenierungen zudem offenbar relativ starken Änderungen unterworfen waren. Dies überrascht nicht, wenn man sich vergegenwärtigt, dass Operninszenierung zur Zeit Meyerbeers ein hochgradig arbeitsteiliges Unternehmen war, das zahlreiche Bereiche umfasste und ebenso viele verantwortliche Entscheidungsträger und Kompetenzen vereinigte. Die Hauptverantwortung lag weiterhin beim Autor, also Eugène Scribe, der sich mit Meyerbeer zu mehreren Konferenzen über die Inszenierung traf und mit diesem die Mise en scèneProben leitete. Die Inszenierung der Uraufführung des Prophète wurde von folgenden Persönlichkeiten bestimmt:

$\begin{array}{ll}\text { Eugène Scribe } & \text { Libretto } \\ \text { Giacomo Meyerbeer } & \text { Musik } \\ \text { Edmond Duponchel } & \text { Directeur } \\ \text { Nestor Roqueplan } & \text { Directeur } \\ \text { Auguste Mabille } & \text { Choreographie } \\ \text { Paolo Taglioni } & \text { Choreographie }\end{array}$




$\begin{array}{ll}\text { Charles Cambon } & \text { Dekorationen } \\ \text { Edouard Despléchin } & \text { Dekorationen } \\ \text { Charles Séchan } & \text { Dekorationen }\end{array}$

Die Frage der schriftlichen Fixierung durch Palianti war dem Inszenierungsprozess selbst zeitlich nachgeordnet. Meyerbeers Briefwechsel gibt über sein gewandeltes Verhältnis zur Inszenierung deutlichen Aufschluss. Zwar stand Meyerbeer auch schon zur Zeit des Robert le Diable und der Hugenotten mit dem Theateragenten Duverger, der die ersten summarischen Szenenanweisungen dieser Werke veröffentlichte, wegen der musikalischen Einrichtung auswärtiger Produktionen in persönlichem Kontakt, es gibt jedoch - zumindest nach meiner Kenntnis - keinerlei Hinweise darauf, dass sie sich jemals in Fragen der Inszenierung ausgetauscht hätten. ${ }^{3 \mathrm{I}}$

Dies ändert sich seit dem Prophète radikal. Mit Louis Palianti stand Meyerbeer insbesondere einige Monate nach der Premiere von Le Prophète am 16. April I 849 in intensivem Austausch. Bereits vier Wochen später, am I 5. Mai, findet sich in Meyerbeers Taschenkalender der Eintrag: »Brandus wegen Palliantin « [sic]. Möglicherweise ging vom Komponisten selbst die Initiative aus, mit Palianti zusammenzutreffen, wofür er sich der Vermittlung seines Verlegers Brandus versicherte..$^{32}$ Drei Monate später taucht der Name Palianti im Taschenkalender wieder unter demjenigen des Verlegers auf: »Brandus. Palianti beeilen und auch Notizen über das Sonnenlicht und Schlittschuhe geben. «33 Demnach war es offenbar Meyerbeer selbst, der dem Theateragenten die technischen Informationen für die Anfertigung der Rollschuhe und vor allem für das elektrische Licht der so genannten »Prophetensonne« lieferte, oder der zumindest seinen Verleger hierzu beauftragte.

Mehr als einmal musste Meyerbeer den säumigen Palianti ermahnen, die Redaktion und Veröffentlichung des Livret de mise en scène zu beschleunigen. Am 2 I. August schrieb er diesbezüglich aus Bad Gastein an Brandus:

»Es wäre wegen der Provinztheater, und auch wegen der deutschen Theater sehr wünschenswerth wenn Palianti die Publikation der mise en Scène beschleunigte. Ebenfall's wäre es gut wenn er am Schluß der mise en Scène einige Indicationen gäbe über die Art und Weise wie das Sonnenlicht im 3 ten Akt hervorgebracht wird, und den Namen und die Adresse des Mannes beifügte bei welchem man die dazu nöthige Vorrichtung kaufen kann. (Von Duponchel kann er den Namen und die Adresse des Erfinders erfah-

${ }^{31}$ Aus Meyerbeers Briefen und Tagebüchern wissen wir, dass sich der Komponist mit Duverger während der Zeit von Robert le Diable und Les Huguenots mehrfach traf. Vgl. BwTb II, I74-175, 275 .

${ }_{32}$ BwTb IV, 490.

$33 \mathrm{Bw} \mathrm{Tb} \mathrm{V}, 4 \mathrm{I}$. 
ren.) Deßgleichen sollte er eine Indication geben wie die Schlittschuhe gemacht sind. Ich bin über diese beiden Gegenstände in wenigstens 6 Theatern befragt worden. «34

Am II. Oktober I849, fast ein halbes Jahr nach der Uraufführung des Prophète, korrigierte Meyerbeer die Anweisungen Paliantis und formulierte Kommentare zu ihnen: »Ich übergab Palianti seine Mise en scène nebst meinen Bemerkungen, u. wir diskutierten sie. «35 Zwei Tage später trafen sich Palianti und Meyerbeer erneut zu einer abschließenden Besprechung. Am 25. Oktober findet sich im Taschenkalender der bemerkenswerte Eintrag »an Brandus Geschenk an Palianti «, der darauf hindeuten könnte, dass Meyerbeer entweder mit Paliantis Arbeit sehr zufrieden war oder aber dass er den Theateragenten zu weiteren Anstrengungen bei der Publikation der Inszenierungsanweisungen zu Le Prophète anspornen wollte. ${ }^{36}$ Erneut begegnet ein nicht näher erläuterter Vermerk »an Palianti« am 4. November im Taschenkalender.

Im November I 849 schließlich erschien Paliantis Mise en scène $\mathrm{zu}$ Le Prophète im Supplément der Revue et Gazette des Théâtres. Nach den geschilderten Dokumenten ist anzunehmen, dass die außergewöhnliche Detailgenauigkeit von Paliantis Anweisungen nur durch die intensiven Konferenzen, die Meyerbeer und der Theateragent hierüber führten, erklärt werden kann. Zugleich ist festzuhalten, dass Palianti persönlich nicht das geringste mit der konkreten szenischen Realisierung des Werkes zu tun hatte. Es handelt sich also ganz eindeutig um eine ausschließlich deskriptive und keineswegs um eine präskriptive Inszenierungsanweisung.

Ein ähnlicher Ablauf in der Zusammenarbeit zwischen Meyerbeer und Palianti wiederholte sich anlässlich der Premiere von L'Étoile $d u$ Nord (I6. Februar I 854). Das erste dokumentierte Treffen zwischen beiden fand etwa sechs Wochen nach der Uraufführung am 27. März statt. ${ }^{37}$ Eine weitere Begegnung folgte am I. April: "Palianti lieset mir die Mise en scène de l'Etoile du Nord vor, welche er für die Bühnen drucken lässt. «38

Dass Meyerbeer auch persönlich die Distribution der Inszenierungen vornahm, geht aus einem Tagebucheintrag vom 8 . Mai i 854 hervor:

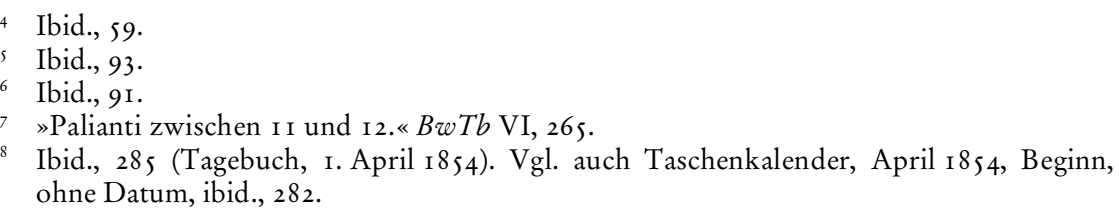


»Brief von August Lewald aus Stuttgart: verlangt L'Etoile zur Aufführung in Stuttgart. An Lewald die Mise en scène von Palianti und das Libretto der Etoile du Nord geschickt. «39

Bereits elf Tage später gab Lewald dem Stuttgarter Generalintendanten Ferdinand Freiherr von Gall einen ausführlichen Bericht über die Vorbereitungen der Stuttgarter Produktion, wobei auch Paliantis Name fällt:

»Kosten wird die Ausstattung nicht viel, und besetzen können wir sie auch. Wir besetzen ja Alles und es ist caprice zu sagen, die Marketenderinnen sind nicht zu besetzen, wenn gleich zugestanden werden muß, daß sie es hier sehr schön machen und daß es nicht möglich ist, wenn man Herrn Palliantis Mise en scène noch so gut studiert hat, es andern Sängerinnen klar zu machen, wie das gegeben werden muß, wenn man es nicht mehrmals hier gesehen und zwar mit Regisseur Augen gesehen hat. « ${ }^{40}$

In einem Brief vom I6. Oktober I 854 an Georges Hainl informierte Meyerbeer den Dirigenten des Lyoner Opernhauses über zahlreiche Einzelheiten des neuen Werkes, darunter auch über das im II. Akt erforderte Zelt:

«La tente est également d'une très grande importance scénique; sa construction et sa disposition offrent de très grandes difficultés. J'engage donc le théâtre du Grand Opéra de Lyon à se procurer le modèle en petit que M. Palianti a fait faire de cette tente et dont on fait usage tous les théâtres d'Allemagne qui ont donné ou se proposent de donner cet ouvrage. $»^{4}$

Eine ähnliche Auskunft erteilte Meyerbeer am 8. November I 854 auch an den Wiener Operndirektor Julius Cornet:

»Obgleich noch nicht hergestellt, benutze ich den Moment wo ich wenigstens im Stande bin eine Antwort zu dictiren, um Ihnen mitzutheilen daß ich, ihrem Wunsche gemäß, H Pallianti in Paris in Ihrem Namen ersucht habe, Ihnen das kleine Modell des Zeltes zu schicken, und wird dasselbe hoffentlich schon in Ihren Händen sein. $\ll^{42}$

Anstelle eines Fazits möchte ich nochmals auf die eingangs aufgeworfene Frage der Konservierung werkhafter Inszenierungen zu sprechen kommen. Dabei stellt sich zunächst das Problem der Autorschaft der Regiebücher. Diese wurden im Falle der Grand Opéra, mit Ausnahme der Muette, grundsätzlich von Theateragenten erstellt, die nichts mit dem Produktionsprozess selbst zu tun hatten. Inszenierung im I9. Jahrhundert war eine hochgradig arbeitsteilige und ständigen Veränderungen unterworfene Gemeinschaftsarbeit, die nicht von

39 Ibid., 305 .

40 Ibid., 780 .

$4^{1} \quad$ Ibid., 406.

$4^{2}$ Ibid., 4I3. 
vornherein in allen Details schriftlich festgelegt, sondern vielmehr erst nachträglich zu bestimmten Zeitpunkten protokolliert wurde.

Auch Meyerbeer agierte völlig im Einklang mit dem Produktionssystem seiner Zeit, wenn er - dem Zeugnis Emile Perrins zufolge - genau wie seine Pariser Zeitgenossen Auber oder Halévy der Inszenierung größte Aufmerksamkeit widmete:

«J'ai donc été témoin, depuis plus de trente ans, témoin presque quotidien de ce travail si intéressant et si curieux de la mise en œuvre d'une pièce de théâtre. ... Que ces hommes s'appellent Scribe, Meyerbeer, Auber, Halévy, Verdi, Ambroise Thomas, Ludovic Halévy, je les ai tous vus, animés du même souci, donner à la mise en scène de leurs ouvrages une importance capitale, la considérer comme indispensable à l'expression complète de leur pensée. $»^{43}$

Meyerbeers Streben nach einer möglichst vollständigen Kontrolle des szenischen Ablaufs auch aller späteren und auswärtigen Aufführungen seiner Werke durch verbindliche schriftliche Anweisungen wurde sowohl für Verdi wie auch Wagner vorbildlich und bezeichnet im Prophète offenbar ein neues Stadium gegenüber den älteren Werken der Grand Opéra. Dennoch wäre es anachronistisch anzunehmen, er habe die Inszenierung für alle Ewigkeit verbindlich festlegen wollen. Ebenso unvollkommen wie die szenischen Möglichkeiten seiner Zeit waren auch ihre Aufzeichnungsformen, und in der Bühnenpraxis setzten sich ohnehin bald andere Techniken (wie jene des »durchschossenen Klavierauszugs «) durch, die das Problem der exakten Koordination von Musik und Szene besser zu lösen vermochten.

Die Permanenz einer Produktion im Theaterbetrieb sichert ihrer Realisierung in den einzelnen Vorstellungen eine nur relative Stabilität. Zweifellos gab es schon aus ökonomischen Gründen starke Tendenzen zur Wahrung des einmal Geschaffenen. Gleichzeitig ist unstrittig, wie stark auch Text und Musik im Laufe der Zeit Änderungen unterlagen. Erst Recht gilt dies für die Inszenierung, die durch permanenten technischen Fortschritt sowie ästhetischen, gesellschaftlichen und politischen Wandel geprägt wurde. Sich dieser Einsicht zu verweigern, konnte mitunter groteske Folgen haben. Man denke nur etwa an das reaktionäre Bayreuth der späten I920er-Jahre, wo man auf Anordnung Cosima Wagners noch immer an den völlig antiquierten Leinwandkulissen festhielt, auf denen noch »das Auge des Meisters geruht« hatte - ein Beispiel dafür, wie schnell eine einstmals avantgardistische Bühnenvision in ihr Gegenteil umschlagen kann, denn die ästhetische Halbwertszeit von Dekorationen ist zumeist weitaus geringer als ihre materielle. Mit den zensurbedingten Meta-

43 Emile Perrin, Etude sur la mise en scène, Paris : Quantin I883, 2 If. 
ARNOLD JACOBSHAGEN

morphosen seiner Opern in auswärtigen Aufführungen vertraut, ging es Meyerbeer offenbar weniger um eine »werkhafte« Inszenierung im Sinne Cosima Wagners als vielmehr um die Sicherstellung einer möglichst »werkgerechten « Inszenierung - ein sehr verständliches Anliegen, dessen Durchsetzung in der damaligen Zeit freilich ebenso wenig selbstverständlich war wie heute. 